\title{
Qual o papel dos inibidores da $5 \alpha$-redutase no tratamento da alopecia androgenética? Uma revisão baseada na evidência
}

Leonor Grijó, $^{1}$ Sofia Cardoso, ${ }^{2}$ Liliana Beirão $^{1}$

\section{RESUMO}

Introdução: A alopecia androgenética é a forma mais comum de alopecia. Para o seu surgimento contribuem tanto fatores genéticos como hormonais - estes últimos mediados pela diidrotestosterona (DHT), formada a partir da testosterona numa reação catalisada pela enzima $5 \alpha$-redutase. Alguns estudos têm demonstrado benefício dos inibidores da $5 \alpha$-redutase no tratamento da alopecia androgenética.

Objetivo: Determinar a evidência do efeito dos inibidores da $5 \alpha$-redutase no tratamento da alopecia androgenética em indivíduos adultos.

Métodos: Em março de 2019 foi realizada uma pesquisa bibliográfica de normas de orientação clínica (NOC), meta-análises (MA), revisões sistemáticas (RS) e ensaios clínicos aleatorizados (ECA) na MEDLINE e em sites de medicina baseada na evidência, publicados entre 01/01/2009 e 08/03/2019, em língua portuguesa, inglesa e espanhola, utilizando os termos MeSH Alopecia e 5-alpha reductase inhibitors. Para a atribuição de força de recomendação (FR) e de níveis de evidência (NE) foi utilizada a escala Strengh of Recomendation Taxonomy (SORT), da American Family Physician.

Resultados: Obtiveram-se 91 artigos, dos quais cinco cumpriam os critérios de inclusão: quatro MA e uma RS. Globalmente os estudos parecem mostrar que os inibidores da $5 \alpha$-redutase são eficazes no tratamento da alopecia, sendo de uma forma geral superiores ao placebo.

Conclusões: Conclui-se os inibidores da $5 \alpha$-redutase poderão ser efetivos no tratamento da alopecia androgenética e com uma FR B (SORT). ECA futuros são necessários para esclarecer se existe um fármaco preferencial dentro desta classe, bem como a dose e duração que permita ao indivíduo ter o maior benefício com o menor risco de efeitos adversos. É necessário ainda clarificar se o benefício da terapêutica é igual nos dois sexos.

Palavras-chave: Alopecia; Inibidores da $5 \alpha$-redutase.

\section{INTRODUÇÃO}

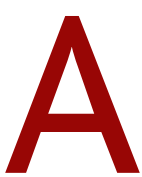
alopecia androgenética é a forma mais comum de alopecia em ambos os sexos, tendo uma prevalência estimada de $50 \%$ nos homens e de $19 \%$ nas mulheres de raça caucasiana. ${ }^{1-2}$ Inicia-se geralmente após a puberdade e é caracterizada por um encurtamento da fase anagénica do ciclo folicular, levando à produção de cabelos mais curtos e mais finos - um processo chamado miniaturi-

1. Médica Interna de Medicina Geral e Familiar. USF Espaço Saúde, ACeS Porto Ocidental.

2. Médica Interna de Medicina Geral e Familiar. USF Maresia, ULS Matosinhos. zação folicular, que conduz à perda progressiva de cabelo terminal. ${ }^{3}$ Apesar do mecanismo patogénico ser semelhante em ambos os sexos, o seu fenótipo é diferente nos homens e mulheres: nos primeiros a perda de cabelo é mais proeminente nas regiões do vértex e frontotemporal; nas mulheres a linha frontal é geralmente poupada, com perda de cabelo apical difusa, manifestada predominantemente por um alargamento na região central. ${ }^{4}$

Para o surgimento da alopecia androgenética contribuem tanto fatores genéticos como hormonais, estes últimos mediados pela DHT, um metabolito da 
testosterona, formado através de uma reação catalisada pela enzima $5 \alpha$-redutase - presente, não só na pele, como também nas glândulas sebáceas e nos folículos capilares do couro cabeludo. Pacientes com alopecia androgenética apresentam níveis mais elevados de $5 \alpha$-redutase, maior produção de DHT e maior número de recetores androgénicos no couro cabeludo calvo. ${ }^{3}$

Sem tratamento, os pacientes sofrem uma perda progressiva de cabelo, levando por vezes a efeitos muito negativos a nível psicológico, com grande impacto na sua autoestima. $^{5}$

\section{Objetivo}

Esta revisão pretende determinar a evidência existente acerca do efeito dos fármacos inibidores da $5 \alpha$-redutase no tratamento da alopecia androgenética em indivíduos adultos.

\section{MÉTODOS}

Em março de 2019 foi realizada uma pesquisa de normas de orientação clínica (NOC), meta-análises (MA), revisões sistemáticas (RS) e ensaios clínicos aleatorizados (ECA) na MEDLINE e em sites de medicina baseada na evidência (Cochrane Library, DARE, Bandolier, Guidelines Finder da National Electronic Library for Health no NHS, National Guideline Clearinghouse, Clinical Practice Guidelines Infobase da Canadian Medical Association), publicados entre 1 de janeiro de 2009 e 8 de março de 2019, em língua portuguesa, inglesa e espanhola, utilizando os seguintes termos MeSH: Alopecia e 5-alpha reductase inhibitors.

Foram incluídos estudos realizados na população adulta (idade $\geq 18$ anos) com alopecia androgenética (população). Os estudos incluídos deveriam ter um grupo exposto à terapêutica com inibidores da $5 \alpha$-redutase (intervenção), comparativamente a um grupo placebo/ausência de tratamento (comparador). O resultado medido (outcome primário) foi o efeito dos fármacos inibidores da $5 \alpha$-redutase na alopecia, nomeadamente em relação à contagem de cabelo, densidade capilar $\left(\right.$ por $\mathrm{cm}^{2}$ ), avaliação fotográfica, autorrelato de melhoria e/ou avaliação de melhoria pelo investigador. Nos artigos que incluíam avaliação de efeitos secundários da terapêutica, este resultado foi também analisado (outcome secundário).

Foram excluídos estudos com outros tipos de alope- cia que não a androgenética; sob terapêutica para a alopecia com outras classes farmacológicas ou derivados botânicos com ação inibidora da $5 \alpha$-redutase, como Serenoa Repens, ou ainda estudos que não tinham grupo placebo como comparador. Foram também excluídos artigos de opinião, editoriais, letters e notícias. Por último, excluíram-se ainda estudos não aleatorizados ou realizados em animais, bem como estudos duplicados.

A seleção dos trabalhos pelo título e resumo foi efetuada por todas as autoras. Os artigos selecionados para leitura integral foram lidos por, pelo menos, duas das autoras para decidir a sua inclusão, em caso de dúvida.

A avaliação final da qualidade e nível de evidência dos artigos incluídos foi discutida e decidida por consenso entre todas as autoras. O nível de evidência (NE) e a força de recomendação (FR) dos artigos, quando ausentes nos artigos originais, foram atribuídos pelas autoras, de acordo com os critérios da escala Strength of Recommendation Taxonomy (SORT), da American Family Physician.

\section{RESULTADOS}

Utilizando os termos de pesquisa nas bases de dados previamente indicadas foram encontrados 91 artigos, dos quais 63 foram excluídos por leitura dos títulos. Destes, oito artigos foram excluídos por repetição. Os restantes foram avaliados por leitura dos resumos, tendo sido excluídos mais sete, resultando um total de 13 artigos para avaliação por leitura integral. Desta leitura resultou a exclusão de mais oito artigos porque o tema e objetivos eram díspares do pretendido e/ou por não estarem de acordo com os critérios de inclusão definidos. O fluxograma de seleção dos estudos está representado na Figura 1.

No final obtiveram-se cinco artigos integrados e avaliados pelas autoras nesta revisão: quatro MA e uma RS. O resumo do corpo de evidência encontra-se explanado nas Quadros I a V.

\section{Meta-análises}

\subsection{Adil e colaboradores (2017)}

A MA de Adil e colaboradores ${ }^{6}$ (Quadro I) incluiu quatro ECA que mostraram que a finasterida na dose de $1 \mathrm{mg}$ /dia é eficaz na promoção do crescimento capilar em homens com alopecia androgenética 


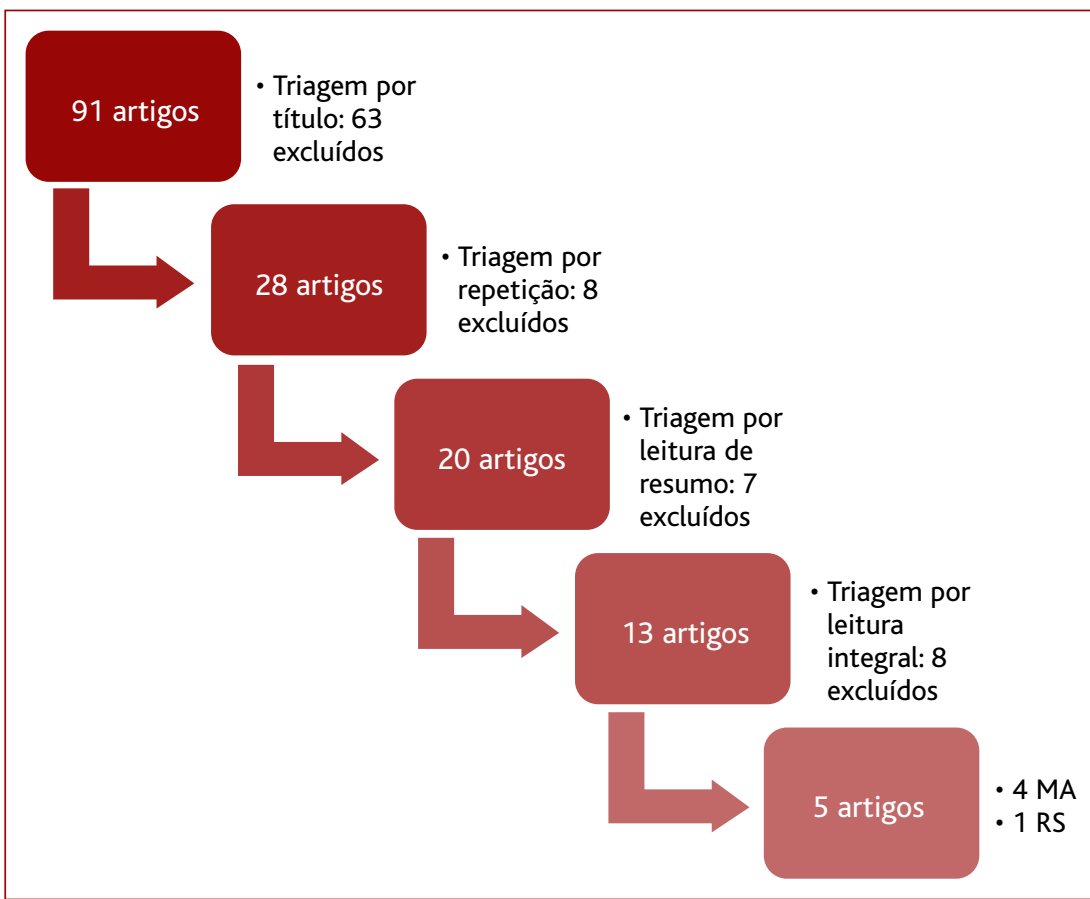

Figura 1. Fluxograma de seleção dos estudos.

(MD=18,37; IC95\%, 9,64-27,11; $p=0,00001)$. O outcome primário avaliado na MA foi a alteração da densidade capilar por $\mathrm{cm}^{2}$. Trata-se de quatro ECA de elevada qualidade, claros quanto à distribuição por grupos, comparação com placebo e dupla-ocultação, resultados consistentes, intenção de tratar e follow up adequado - para serem incluídos na MA os artigos tinham de pontuar como Razoável ou Bom segundo os critérios da U.S. Preventive Services Task Force Quality Rating Criteria. Assim, poderá atribuir-se NE 1 pela escala SORT.

Os autores desta MA fizeram também uma revisão sistemática (RS) de quatro ECA de boa qualidade, com populações e duração semelhantes em todos os estudos, em que foi administrada dutasterida na dose de $0,5 \mathrm{mg} /$ dia e se verificou que esta foi sempre superior ao placebo.

Não foram reportados efeitos adversos graves, embora um grupo pequeno de participantes tenha reportado redução da líbido com a finasterida e dutasterida.

\subsection{Gupta e Charrette (2013) ${ }^{7}$}

A MA de Gupta e Charrette ${ }^{7}$ incluiu 16 ECA que avaliavam a eficácia dos inibidores da $5 \alpha$-redutase finasterida na dose de $1 \mathrm{mg}, 5 \mathrm{mg}$ ou dutasterida na dose de 0,5mg no tratamento da alopecia androgenética, usando os outcomes contagem de cabelo, avaliação fotográfica e relato de melhoria da alopecia por parte dos participantes. Nos estudos que analisavam este parâmetro, os benefícios do tratamento versus risco de efeitos adversos foram também incluídos nos resultados. Tratam-se de 16 ECA de moderada qualidade, com tamanho adequado, clareza quanto à distribuição por grupos, comparação com placebo, resultados consistentes e intenção de tratar. Não há, no entanto, referência acerca das perdas de seguimento nem da forma de ocultação, o que em conjunto com as diferenças verificadas no tempo de intervenção e a heterogeneidade dos tamanhos amostrais leva à atribuição de um NE 2 a esta RS.

No que respeita à contagem de cabelo, a diferença média entre o placebo e dutasterida na dose de $0,5 \mathrm{mg}$ foi OR=10, 12 (IC95\%, 4,54-15,69; $n=552$ ); finasterida na dose de $1 \mathrm{mg}$ OR=8,64 (IC95\%, 7,57-9,7; $n=3.210$ ) e finasterida na dose de $5 \mathrm{mg} \mathrm{OR}=12,35$ (IC95\%, 7,26-17,43; $n=282$ ), revelando que todos os inibidores da $5 \alpha$-redutase estudados foram significativamente mais eficazes que o placebo. Em relação ao outcome avaliação fotográfica todos os fármacos nas dosagens estudadas foram significativamente superiores ao placebo, não havendo diferença significativa entre eles. No que se refere ao relato de melhoria da alopecia por parte dos participantes, os fármacos não diferiram significativamente, sendo neste ponto apenas a finasterida na dose de $1 \mathrm{mg}$ mais eficaz que o placebo. Por último, em relação a eventos adversos não houve diferença significativa em relação ao placebo, reforçando a segurança dos fármacos analisados.

\subsection{Olsen e colaboradores (2012)}

A MA de Olsen e colaboradores ${ }^{8}$ (Quadro III) incluiu três ECA, de razoável qualidade metodológica, claros quanto à distribuição por grupos, ocultação, intenção de tratar e tamanho da população, embora com 


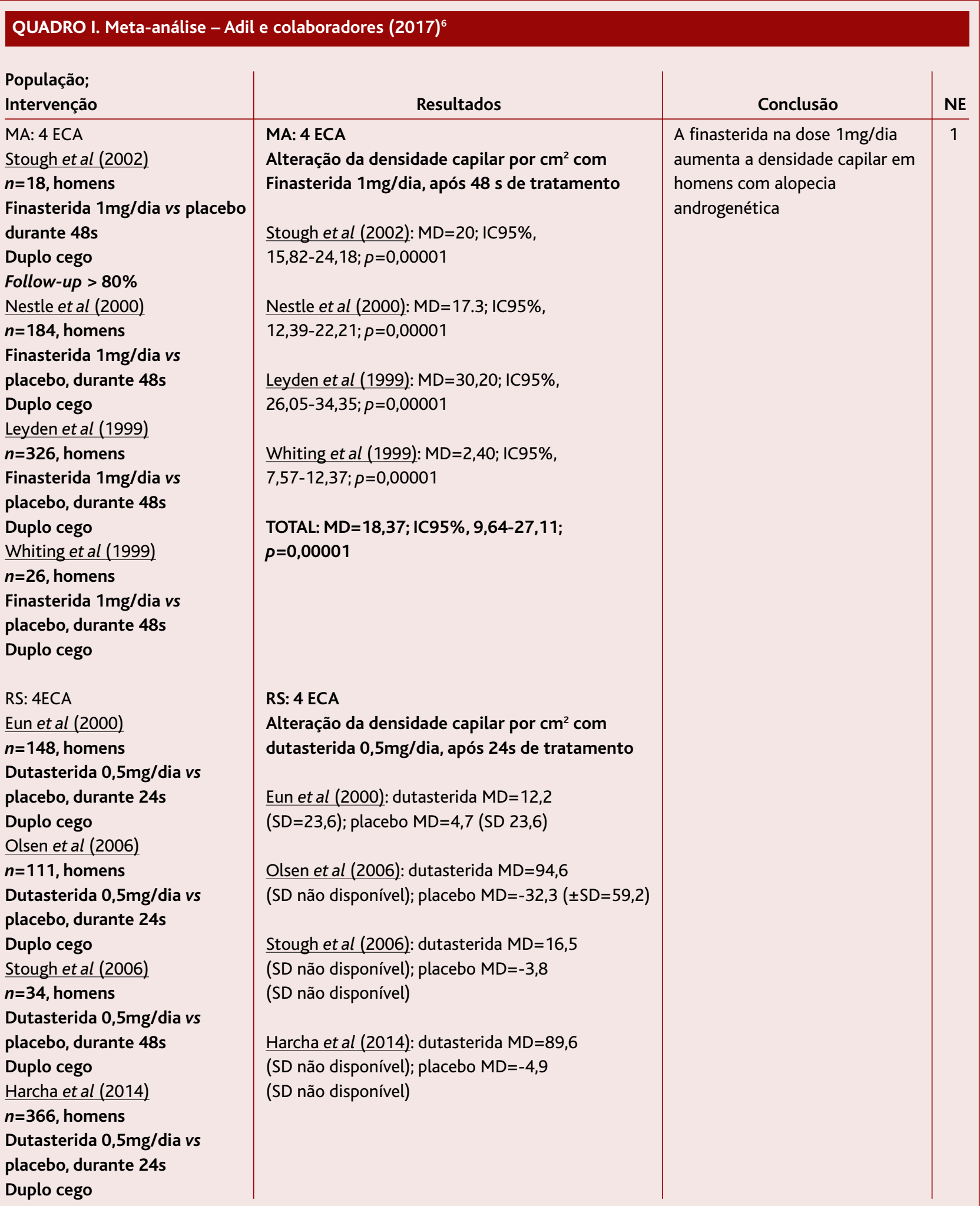




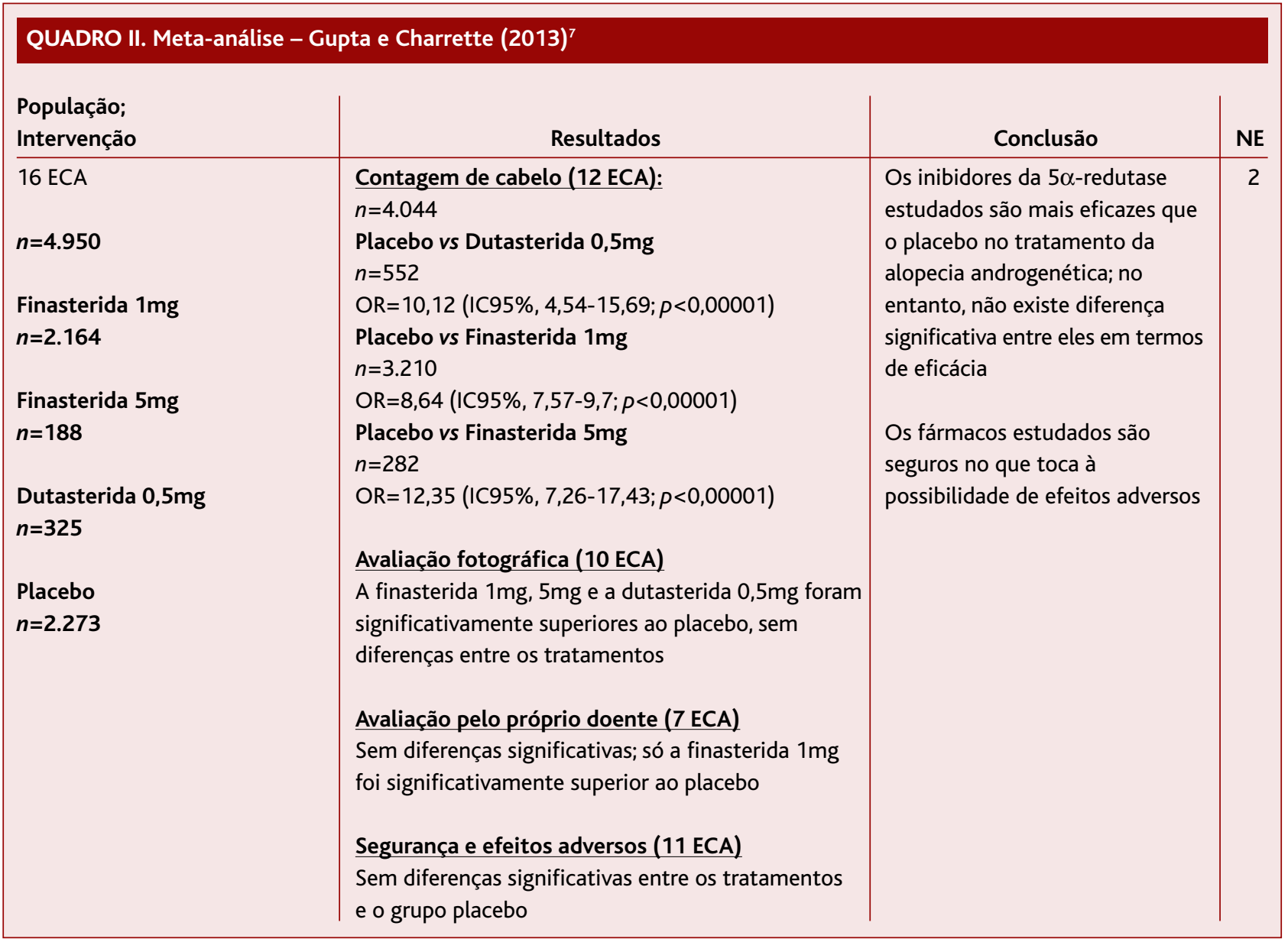

follow-up inadequado. Os dois primeiros ECA (Kaufman e colaboradores e Finasteride Male Pattern Hair Loss Study Group) são estudos replicados, pelo que os dados são apresentados agrupadamente. A duração dos três ECA foi de dois anos, embora com populações de idades diferentes: dois com homens com idades entre os 18 e os 41 anos e um com homens com idades entre os 41 e os 60 anos. Nos três ECA foi administrada finasterida per os na dose de $1 \mathrm{mg} /$ dia e a sua eficácia foi avaliada através de fotografias clínicas estandardizadas do vértex, região craniana anterior/média, frontal e temporal ao longo de 24 meses. Aos 24 meses, o tratamento resultou num crescimento de cabelo estatisticamente significativo em todas as zonas do crânio nos homens 18-41 anos e no vértex e zona anterior e média craniana nos homens com idades entre os 41-60 $(p<0,001)$. Já nas regiões frontal e temporal, nos ho- mens entre 41-60 anos o efeito foi bastante menor, apesar de estatisticamente significativo $(p \leq 0,05)$.

\subsection{Mella e colaboradores (2010)}

MA constituída por 12 ECA ( $n=3.927$ ) que compararam a finasterida na dose de 1 ou $5 \mathrm{mg}$ com o placebo no tratamento de adultos com alopecia androgenética em relação aos seguintes outcomes: relato de melhoria da alopecia por parte dos participantes, contagem de cabelo, classificação de melhoria da alopecia por parte dos investigadores, avaliação fotográfica e efeitos adversos a curto (12 meses) e a longo prazo (24 meses).

Os ECA incluídos nesta MA são claros quanto à distribuição por grupos, intenção de tratar, comparação com placebo e apresentam na sua maioria dupla-ocultação, follow-up e tamanho adequados. A inconsistência de alguns dos resultados e a possível existência de 


\begin{tabular}{|c|c|c|c|}
\hline $\begin{array}{l}\text { População; } \\
\text { Intervenção }\end{array}$ & Resultados & Conclusão & NE \\
\hline $\begin{array}{l}3 \text { ECA: } \\
\frac{2 \text { ECA }}{n=1.553} \\
\text { Finasterida } 1 \mathrm{mg} / \text { dia vs } \\
\text { placebo, durante } 24 \text { meses } \\
\text { Duplo cego } \\
\text { Follow-up < 80\% } \\
\\
\text { Whiting et al } \\
n=424 \\
\text { Finasterida } 1 \mathrm{mg} / \text { dia vs } \\
\text { placebo, durante } 24 \text { meses } \\
\text { Duplo cego } \\
\text { Follow-up }>80 \%\end{array}$ & $\begin{array}{l}\text { - Homens } 18-41 \text { anos - crescimento de cabelo } \\
\text { estatisticamente significativo }(p<0,001) \text { em todas } \\
\text { as zonas do crânio } \\
\text { - Homens } 41-60 \text { anos - crescimento de cabelo } \\
\text { estatisticamente significativo }(p<0,001) \text { no vértex e } \\
\text { zona anterior e media craniana } \\
\text { - Regiões frontal e temporal crescimento menor de } \\
\text { cabelo apesar de estatisticamente significativo } \\
(p \leq 0,05)\end{array}$ & $\begin{array}{l}\text { A finasterida ( } 1 \mathrm{mg} \text { ) pareceu ser } \\
\text { efetiva na redução da alopecia } \\
\text { em todas as zonas cranianas. Os } \\
\text { efeitos são maiores nas zonas do } \\
\text { vértex e região craniana anterior/ } \\
\text { /média, com maior eficácia nos } \\
\text { homens mais jovens quando } \\
\text { comparado com homens mais } \\
\text { velhos. }\end{array}$ & 2 \\
\hline
\end{tabular}

viés de publicação leva à atribuição de um NE 2 a esta RS.

A proporção de doentes que relataram uma melhoria foi maior com a finasterida do que com o placebo, tanto a curto (RR=1,81; IC95\%, 1,42-2,32) como a longo prazo ( $R R=1,71$; IC95\%, 1,15-2,53), em ensaios com evidência de moderada qualidade. Adicionalmente, o fármaco demonstrou aumentar a contagem capilar em comparação com o placebo (expressa pela percentagem da contagem inicial em cada indivíduo), tanto a curto prazo (MD=9,42\%; IC95\%, 7,95-10,9\%) como a longo prazo (MD=24,3\%; IC95\%, 17,92-30,60\%), em estudos de moderada qualidade. A proporção de doentes classificados como melhorados por parte dos investigadores foi maior a curto prazo $(\mathrm{RR}=1,80$; IC95\%, 1,43$2,26)$, evidência de moderada qualidade. No que respeita a efeitos adversos, evidência de moderada qualidade sugere um aumento do risco de disfunção eréctil ( $R R=2,22$; IC95\%, 1,03-4,78) e um possível aumento no risco de outras complicações sexuais $(\mathrm{RR}=1,39$; IC95\%, 0,99-1,95); no entanto, o risco de descontinuação do tratamento por efeitos adversos demonstrou ser semelhante ao placebo (RR=0,88; IC95\%, 0,51-1,49), evidência de moderada qualidade.

\section{Revisão sistemática}

\subsection{Van Zuuren e colaboradores (2016) $^{10}$}

O objetivo desta revisão sistemática foi o de determinar a eficácia e segurança de diferentes opções para o tratamento da alopecia androgenética em mulheres. Foram para isso analisados 47 ECA que incluíram um grande número de intervenções, com um total de 5.290 participantes; no entanto, apenas três desses estudos ( $n=231$ ) incluíram um inibidor da $5 \alpha$-redutase comparado com placebo; neste caso, a finasterida na dose de $1 \mathrm{mg}$ uma vez por dia. Tratam-se de ECA de moderada qualidade, claros em relação à distribuição por grupos, intenção de tratar e comparação com placebo. Num dos ECA desta RS, o follow-up foi, no entanto, inadequado e num outro não foi clara a forma de ocultação. Tratam-se ainda de ECA com poucos participantes e heterogéneos em relação ao tempo de seguimento.

Em relação ao relato de melhoria da alopecia por parte dos participantes (no único estudo em que foi avaliado) verificou-se que a finasterida não foi mais eficaz que o placebo (RR=0,95; IC95\%, 0,66-1,37), tendo esse achado sido consistente com as avaliações dos investigadores (RR=0,77; IC95\%, 0,31-1,90). Em relação à contagem capilar, por sua vez, em dois dos estudos (219 


\begin{tabular}{|c|c|c|c|}
\hline $\begin{array}{l}\text { População; } \\
\text { Intervenção }\end{array}$ & Resultados & Conclusão & NE \\
\hline $\begin{array}{l}12 \text { ECA } \\
n=3.927 \\
\text { Finasterida ( } 1 \text { ou } 5 \mathrm{mg} / \text { dia) } \\
n=2.152 \\
\text { vs } \\
\text { placebo } \\
n=1.775\end{array}$ & 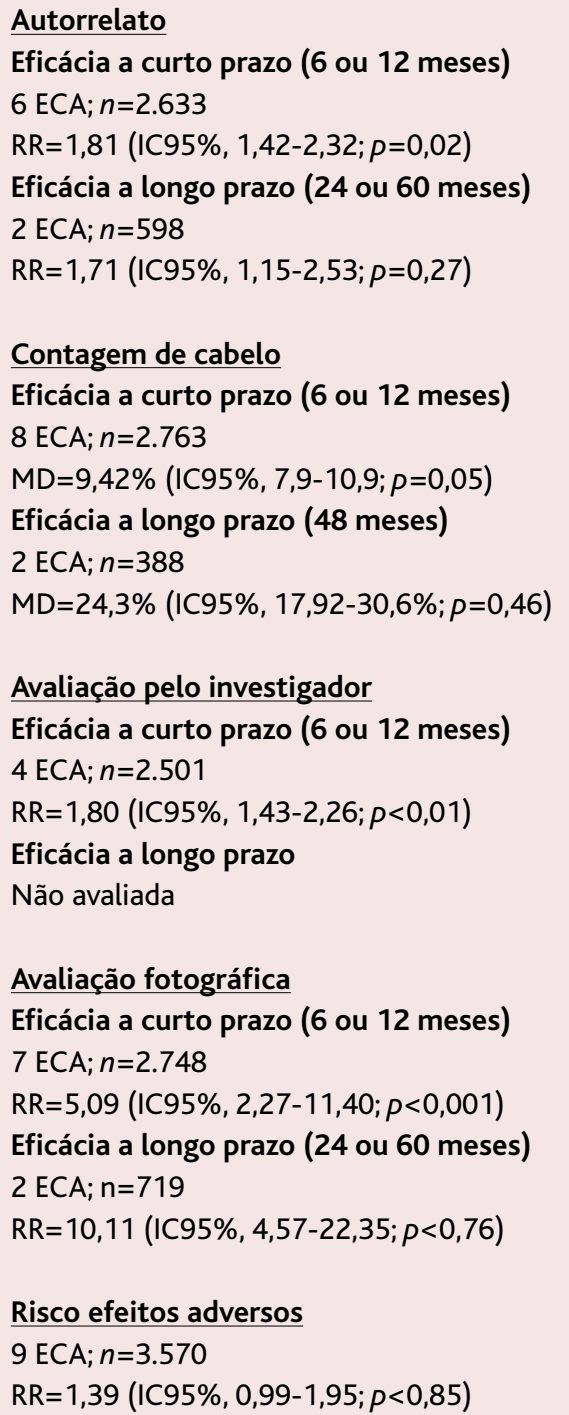 & $\begin{array}{l}\text { O uso diário de finasterida } \\
\text { aumenta as contagens de cabelo } \\
\text { e melhora tanto a avaliação do } \\
\text { utente quanto a do investigador } \\
\text { e com eficácia sustentada ao } \\
\text { longo do tempo, apesar de } \\
\text { aumentar o risco de disfunção } \\
\text { sexual. } \\
\text { O estudo concluiu que não há } \\
\text { diferenças significativas em } \\
\text { termos de outcomes entre as } \\
\text { doses de } 1 \text { e de } 5 \mathrm{mg} \text {. }\end{array}$ & 2 \\
\hline
\end{tabular}

participantes) não houve diferença clinicamente significativa, sendo que neste aspeto apenas um dos estudos analisados (12 participantes) favoreceu a finasterida. Em relação à ocorrência de efeitos adversos, segurança e tolerabilidade, apenas um estudo analisou essa questão, sendo estes similares em ambos os grupos ( $R R=1,03$; IC95\%, 0,45-2,34). Os autores concluem, assim, que a finasterida não foi mais eficaz que o place- bo, sendo necessários mais estudos que analisem diferentes dosagens ou outros fármacos desta classe.

\section{CONCLUSÕES}

De uma forma geral os estudos parecem mostrar que os inibidores da $5 \alpha$-redutase são eficazes na melhoria da alopecia androgenética em indivíduos adultos, sendo sempre superiores ao placebo. A única exceção, em 


\begin{tabular}{|c|c|c|c|}
\hline $\begin{array}{l}\text { População; } \\
\text { Intervenção }\end{array}$ & Resultados & Conclusão & NE \\
\hline $\begin{array}{l}3 \mathrm{ECA} \\
n=231 \text { mulheres adultas com } \\
\text { alopecia androgenética } \\
\text { Intervenção: } \\
\text { Finasterida } 1 \mathrm{mg} / \text { dia vs placebo } \\
\text { Duração: } 6 \text { meses ( } 1 \mathrm{ECA} \text {, } \\
n=13) \text { ou } 1 \text { ano ( } 2 \text { ECA } n=218 \text { ) }\end{array}$ & $\begin{array}{l}\text { Outcomes primários: } \\
\text { - Relato de melhoria da alopecia por parte dos } \\
\text { participantes: RR=0,95; IC95\%, 0,66-1,37; avaliado } \\
\text { apenas num dos ECT ( } n=137) \text {. } \\
\text { - Mudança na qualidade de vida (QoL): nenhum } \\
\text { dos ECA avaliou. } \\
\text { - Efeitos adversos, segurança e tolerabilidade: } \\
\text { RR=1,03; IC95\%, 0,45-2,34; avaliado apenas num } \\
\text { dos ECA ( } n=137) \text {. } \\
\text { Outcomes secundários: } \\
\text { - Proporção de participantes classificados com } \\
\text { melhoria da alopecia por parte dos investigadores: } \\
\text { RR=0,77; IC95\%, } 0,31-1,90 ; \text { avaliado apenas num } \\
\text { dos ECA ( } n=137) \text {. } \\
\text { - Contagem capilar: não estimável; avaliado nos } \\
3 \text { ECA ( } n=231) \text {. }\end{array}$ & $\begin{array}{l}\text { A finasterida não foi mais eficaz } \\
\text { que o placebo. }\end{array}$ & 2 \\
\hline
\end{tabular}

que tal não se verificou, foi na RS de Van Zuuren e colaboradores, ${ }^{10} \mathrm{em}$ que a finasterida na dose de $1 \mathrm{mg}$ por dia não foi mais eficaz que o placebo: tratou-se, no entanto, de uma conclusão retirada de três estudos com poucos participantes, um deles com follow-up inadequado e o único dos estudos incluídos nesta revisão que avaliava o efeito desta classe de fármacos no tratamento da alopecia androgenética em mulheres.

Em relação a efeitos secundários da terapêutica, os estudos que avaliam este parâmetro como outcome secundário concluem de uma forma geral que os inibidores da $5 \alpha$-redutase não estiveram associados a efeitos adversos significativos (reforçando, assim, a sua segurança) e, quando estes ocorreram, nomeadamente no estudo de Mella e colaboradores, ${ }^{9}$ o risco de descontinuação do tratamento devido aos efeitos adversos demonstrou ser semelhante ao placebo.

Verifica-se, todavia, que os ECA realizados nesta área são de uma forma geral pouco recentes, motivo pelo qual (tendo em conta os critérios de exclusão) esta revisão se baseia apenas em MA e RS. São ainda, na sua maioria, heterogéneos em relação ao tempo de exposição, à dose e fármaco administrado e ao tamanho amostral. Verificou-se também que apenas um dos estudos encontrados avaliou o efeito da terapêutica em mulheres com alopecia androgenética. ${ }^{10}$

A evidência é, assim, ainda pouco consistente, sendo importante neste sentido a realização de mais ECA, idealmente com um maior número de participantes $\mathrm{e}$ mais homogéneos (nomeadamente em relação aos tamanhos amostrais, duração de tratamento, doses e fármacos estudados), no sentido de perceber qual a dose e o fármaco preferencial dentro da classe, bem como a duração ideal de tratamento que consiga uma melhor relação risco-benefício nestes doentes. Seria importante ainda a realização de mais ECA em mulheres com alopecia androgenética, a fim de se verificar se o efeito benéfico que aparentemente existe com a utilização destes fármacos em homens se estende às mulheres com o mesmo problema.

De acordo com o corpo de evidências analisado nesta revisão conclui-se que os inibidores da $5 \alpha$-redutase poderão ser efetivos no tratamento da alopecia androgenética e com uma FR B (SORT).

\section{REFERÊNCIAS BIBLIOGRÁFICAS}

1. Krupa SD, Chakravarthi M, Shilpakar R. Male androgenetic alopecia: population-based study in 1,005 subjects. Int J Trichology. 2009;1(2):131-3. 
2. Norwood OT. Incidence of female androgenetic alopecia (female pattern alopecia). Dermatol Surg. 2001;27(1):53-4.

3. Ellis JA, Sinclair R, Harrap SB. Androgenetic alopecia: pathogenesis and potential for therapy. Expert Rev Mol Med. 2002;4(22):1-11.

4. Levy LL, Emer JJ. Female pattern alopecia: current perspectives. Int J Womens Health. 2013;5:541-56.

5. Alfonso M, Richter-Appelt $H$, Tosti A, Viera MS, García M. The psychosocial impact of hair loss among men: a multinational European study. Curr Med Res Opin. 2005;21(11):1829-36.

6. Adil A, Godwin M. The effectiveness of treatments for androgenetic alopecia: a systematic review and meta-analysis. J Am Acad Dermatol. 2017;77(1):136-41.e5.

7. Gupta AK, Charrette A. The efficacy and safety of $5 \alpha$-reductase inhibitors in androgenetic alopecia: a network meta-analysis and benefit-risk assessment of finasteride and dutasteride. J Dermatolog Treat. 2014;25(2):156-61.

8. Olsen EA, Whiting DA, Savin R, Rodgers A, Johnson-Levonas AO, Round $E$, et al. Global photographic assessment of men aged 18 to 60 years with male pattern hair loss receiving finasteride $1 \mathrm{mg}$ or placebo. J Am Acad Dermatol. 2012;67(3):379-86.

9. Mella JM, Perret MC, Manzotti M, Catalano HN, Guyatt G. Efficacy and safety of finasteride therapy for androgenetic alopecia: a systematic review. Arch Dermatol. 2010;146(10):1141-50.

10. Van Zuuren EJ, Fedorowicz Z. Interventions for female pattern hair loss. JAMA Dermatol. 2017;153(3):329-30.

\section{CONFLITO DE INTERESSES}

As autoras declaram não ter quaisquer conflitos de interesse.

\section{ENDEREÇO PARA CORRESPONDÊNCIA}

Leonor Grijó

E-mail: leonor.grijo@gmail.com

https://orcid.org/0000-0002-8222-6120

Recebido em 12-04-2019

Aceite para publicação em 23-07-2019

\section{ABSTRACT}

\section{WHAT IS THE ROLE OF $5 \alpha$-REDUCTASE INHIBITORS IN THE MANAGEMENT OF ANDROGENETIC ALOPECIA?} AN EVIDENCE-BASED REVIEW

Introduction: Androgenetic alopecia is the most frequent form of alopecia. Both genetic and hormonal factors - these last ones mediated by dihydrotestosterone (DHT) (formed from testosterone in a reaction catalyzed by the enzyme $5 \alpha$-reductase) contribute to its onset. Some studies have demonstrated the benefit of $5 \alpha$-reductase inhibitors in the treatment of androgenetic alopecia.

Objective: To determine the evidence of the treatment with $5 \alpha$-reductase inhibitors in adults with androgenetic alopecia. Methods: In March 2019, we did research of evidence-based guidelines, meta-analysis (MA), systematic reviews (RS) and randomized controlled trials (RCTs) in MEDLINE and online evidence-based medicine websites. The search was limited to publications between 01/01/2009 and 08/03/2019, in Portuguese, English, and Spanish, using the MeSH terms 'Alopecia' and '5-alpha reductase inhibitors'. To rate the quality of a study and the strength of a recommendation we used the scale Strength of Recommendation Taxonomy (SORT) of the American Academy of Family Physicians.

Results: In total, 91 potentially relevant papers were identified and 5 met the inclusion criteria: four MA and one RS. Overall, the studies seem to show that $5 \alpha$-reductase inhibitors are effective in the treatment of androgenetic alopecia.

Conclusions: $5 \alpha$-reductase inhibitors may be effective in the treatment of androgenetic alopecia and with a strength of recommendation B (SORT). Future RCT are needed to clarify if there is a preferred drug within this class, as well as the dose and duration that allows the greatest benefit with the least risk of adverse effects. It is also necessary to clarify if the benefits of the therapy are the same in both genders.

Keywords: Alopecia; $5 \alpha$-reductase inhibitors. 